\title{
Jan Priewe
}

\section{Vom Lohnarbeiter zum Shareholder?}

\section{Lohnarbeit im Zeichen des Shareholder-Value - ein Auslaufmodell?}

In den 90er Jahren waren in allen entwickelten Volkswirtschaften die folgenden fünf Trends, freilich in unterschiedlichem Ausmaß, zu beobachten:

- Die Shareholder-Value-Orientierung setzt sich zunehmend durch, zumindest bei großen Aktiengesellschaften; dem Kapitalmarkt kommt im Rahmen der corporate governance eine größere Bedeutung gegenüber anderen Formen der Unternehmenskontrolle zu.

- Eine neue und ausgeprägtere Aktienkultur tauchte auf, die in Deutschland im Lauf der 90er Jahre von einem starken Anstieg der Aktienkurse begleitet war, unterbrochen von gelegentlichen Blasen und deren Platzen. Die Marktkapitalisierung (Börsenwert der Unternehmen) steigt.

- Management, leitende Mitarbeiter und zunehmend auch tariflich entgoltene Arbeitnehmer werden stärker als zuvor am Kapital des arbeitgebenden Unternehmens beteiligt, zudem nehmen auch außer- und überbetriebliche Kapitalbeteiligungen von Arbeitnehmern zu.

- Die Unternehmen drängen immer stärker auf Lohnflexibilisierung: Neben einem festen Grundlohn sollen flexible Entgeltbestandteile vereinbart werden, die gewinnabhängig oder direkt mit Kapitalbeteiligungen verbunden sind (z.B. Aktien oder Aktienoptionen statt Lohn).

- Ein steigender Teil der Altersvorsorge von Arbeitnehmern wird - wie bei Selbständigen - privat durch Kapitalanlagen der verschiedensten Art implementiert. Mithin steigt der Anteil kapitalgedeckter Alterssicherung, nicht zuletzt durch den Rückbau der gesetzlichen Rentenversicherung.

$\mathrm{Ob}$ es sich dabei um quantitativ bedeutsame, anhaltende und nicht durch Gegentendenzen neutralisierte oder überkompensierte Trends handelt, oder um bloße aufgebauschte Behauptungen mit ideologischer Tendenz, ob sie auch nachhaltig in Zukunft zu erwarten sind, all dies bleibt zu prüfen. Wenn diese Tendenzen aber längerfristig relevant sein werden, lassen sich zwei Hypothesen formulieren: 
- Wenn sich größere Teile der Arbeitnehmer am volkswirtschaftlichen Nettovermögen, insbesondere auch am Produktivvermögen, beteiligen, könnte sich die Vermögensverteilung zugunsten der Arbeitnehmer verändern. Dem steht zweierlei entgegen: Orientiert sich das Management stärker und erfolgreich an der Maximierung des Wertes des Eigenkapitals der Unternehmen, werden die Vermögenswerte der Alteigentümer stark ansteigen. Ferner würde durch Optionspläne und ähnliche Formen der Anteil des Managements am Produktivvermögen erhöht. Per saldo könnte sich daher der Anteil der Arbeitnehmer am Produktivvermögen auch verschlechtern (oder konstant bleiben).

- Wenn sich Arbeitnehmer immer stärker am Produktivvermögen beteiligen, auch aus Gründen der Alterssicherung, werden sie immer mehr zu Teilhabern oder Mit-Unternehmern. Es entstünde eine Art Volkskapitalismus, eine Teilhaber-Gesellschaft (Ludwig Erhard), zwar nicht mit egalitärer Vermögensverteilung, aber doch mit Arbeitnehmern als Produktivvermögensbesitzern. Dies hätte zweifellos Konsequenzen für die Tarifpolitik der Gewerkschaften, für die industriellen Beziehungen und nicht zuletzt für den Charakter von „Lohnarbeit“.

Im folgenden wird argumentiert, daß diese Hypothesen auf die vergangene und die absehbare Entwicklung in Deutschland zwar nicht zutreffen, daß aber auch nicht alles beim Alten bleibt. Relevante Strukturverschiebungen sind durchaus erkennbar.

\section{Empirische Angaben zur Vermögensverteilung in Deutschland}

Obwohl die Datenbasis für Vermögensbestände und deren Verteilung mehr als unzulänglich ist, läßt sich die Vermögensverteilung in Deutschland grob abschätzen. Nach Angaben der Deutschen Bundesbank betrug Ende 1997 das private Bruttovermögen (Sach- und Geldvermögen) in Deutschland gut 14 Billionen DM (bewertet zu Marktpreisen bzw. Börsenkursen des genannten Jahres). Zieht man die Verschuldung der privaten Haushalte in Höhe von 1,8 Billionen DM ab, dann ergibt sich ein Reinvermögen von 12,2 Billionen, rund 50\% mehr als 1990 (DB 1999: 47). Je Haushalt lag das Reinvermögen im gesamtdeutschen Durchschnitt 1997 bei 327.000 DM, in Westdeutschland bei $366.000 \mathrm{DM}$, in Ostdeutschland bei nur 152.400 DM. 50\% des Bruttovermögens sind Immobilien, 38\% Geldvermögen, 12\% sog. Gebrauchsvermögen (vom PKW bis zur Waschmaschine). Das Reinvermögen der privaten Haushalte war 1997 gut 5,1 mal so groß wie das laufende verfügbare Einkommen der Haushalte. Mit 75\% liegt der Schuldenstand der privaten Haushalte (Verpflichtungen, überwiegend Baukredite, im Verhältnis zum verfügbaren Einkommen) im internationalen Vergleich recht niedrig. Das Geldvermögen der privaten Haushalte stieg 1990-97 leicht überdurch- 
schnittlich. Je Haushalt lag es 1997 bei 143.100 DM, im Westen betrug es 163.500 DM, im Osten 51.500 DM.

Betrachtet man die Struktur der Geldvermögensbildung der privaten Haushalte im Jahr 1999, dann sticht die dominante Stellung der Anlagen in Versicherungen mit 21\% ins Auge. Aktien und Aktienfonds machten 16,4\% aus, Investmentzertifikate ${ }^{1} 10,5 \%$. Mithin spielt nach wie vor die Beteiligung an Produktivvermögen seitens der privaten Haushalte eine untergeordnete Rolle. Auf Spareinlagen, Sparbriefe und Termineinlagen konzentriert sich immer noch gut ein Viertel des Geldvermögens. 1999 lag das durchschnittliche Nettogeldvermögen jedes privaten Haushalts bei 103.000 DM. Dies entsprach knapp 161\% des verfügbaren Einkommens der privaten Haushalte (DB 2000: 43). Das Wertpapiervermögen lag bei knapp 63.000 DM je Haushalt.

In den 90er Jahren stieg der Aktienbesitz (einschließlich Aktienfonds) der privaten Haushalte rasant an - von rund 272 Mrd. DM 1991 auf fast 1,2 Billionen DM. Freilich kam dies überwiegend durch die Börsenhausse der 90er Jahre zustande, weniger durch Zukäufe von Aktien.

Auch die Zahl der Aktionäre stieg kräftig, im Jahre 2000 waren fast 19\% der über 14-jährigen Bevölkerung Besitzer von Aktien, Aktienfonds oder Investmentfonds (vgl. DAI 2000, 2001). Der direkte Aktienbesitz wird freilich nur von knapp 10\% der über 14-Jährigen präferiert. Lediglich 2,5\% dieser Bevölkerungsgruppe besitzt Belegschaftsaktien (rund 1,6 Mio Personen), wobei sich nur eine leicht steigende Tendenz herausschälte. 14\% der Arbeitnehmerhaushalte besaßen 1993 Aktien oder Fondsanteile (11\% der Arbeiterhaushalte, $18 \%$ der Angestelltenhaushalte), bei den Selbständigen-Haushalten waren es 16\%. Das Aktienvermögen je Haushalt betrug 1993 knapp 25.000 DM, bei den Arbeitnehmern rund 16.000 DM, bei den Selbständigen 51.000 DM (Bundesregierung 1996).

Die Aktionärsquote in den USA und in Großbritannien, aber auch in den meisten kleineren EU-Ländern ist größer als in Deutschland (in den USA lag die Quote 1998 dreimal so hoch wie in Deutschland). Zu berücksichtigen ist dabei die mit gut 800 geringe Zahl an börsennotierten Aktiengesellschaften in Deutschland - in den USA sind es 7.600, in Großbritannien 2.400. Die Börsenkapitalisierung in den USA beträgt 207\% des BIP, in Deutschland nur 60\%, noch weniger als in Frankreich (diese Zahlen beziehen sich auf Mitte 1999, ISA Consult 2000: 31). ${ }^{2}$ Dies ist vor allem Ausdruck der starken „Mit-

1 Investmentzertifikate können Aktien und Rentenpapiere beinhalten. Die statistischen Angaben folgen den Kategorien des Deutschen Aktieninstituts.

2 Gemessen am BIP war Ende 1996 die Börsenkapitalisierung in den USA noch 4,5 mal so stark wie in Deutschland, in Großbritannien 5,6 mal, in der Schweiz 5 mal, in Schweden 3,8 mal, in den Niederlanden 3,4 mal und in Kanada 3,2 mal so groß wie hierzulande. Nur wenige OECD-Länder hatten eine niedrigere Börsenkapitalisierung als Deutschland (vgl. DB 1997: 28). 1996 gab es unter den 3 Mio. umsatzsteuerpflichtigen Unternehmen in Deutschland nur 3.900 Aktiengesellschaften, von denen nur ein Sechstel an der Börse notiert war. 


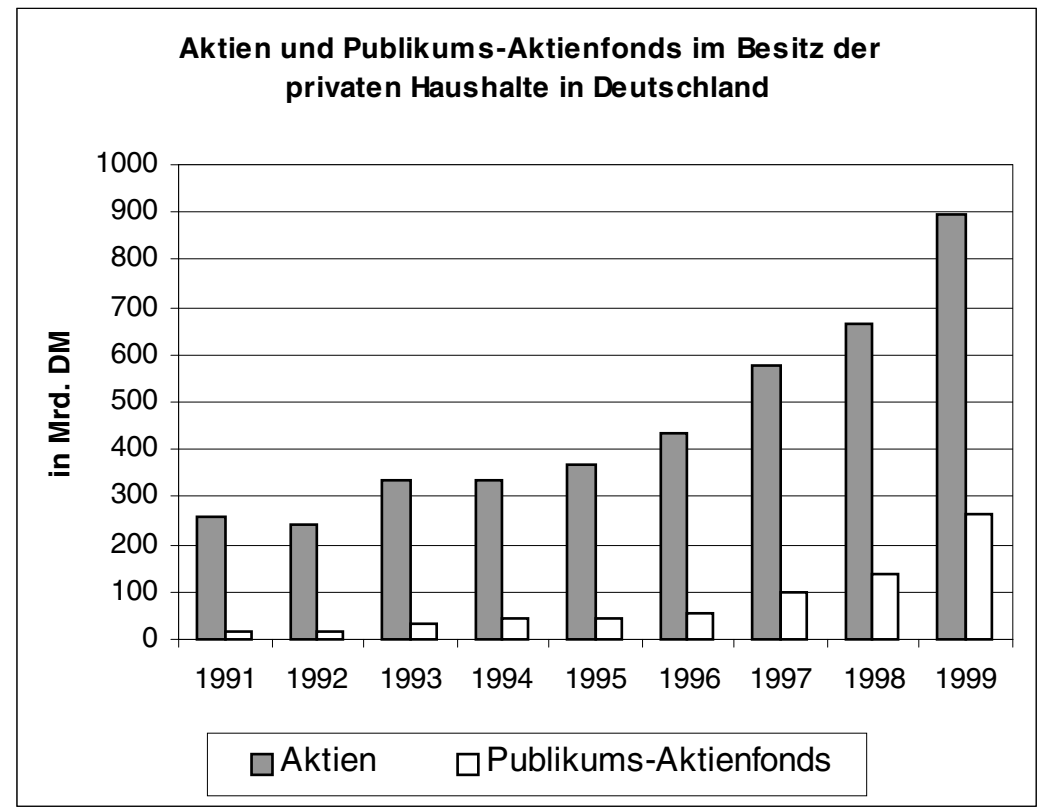

Quelle: DAI 2000: 1

DAX-Entwicklung 1980-2001

(Index: $1987=1000)$

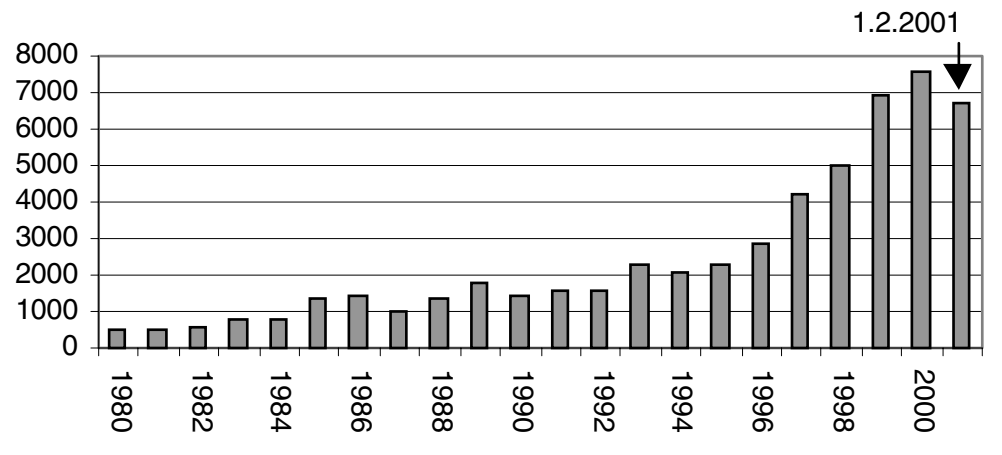

Quelle: SVR 2000, FAZ vom 2.1.2001

telstandskultur“, die in Deutschland zu einem großen Gewicht der Personengsellschaften und $\mathrm{GmbHs}$ führte.

Trotz steigenden Aktienbesitzes der privaten Haushalte sinkt der Anteil der 


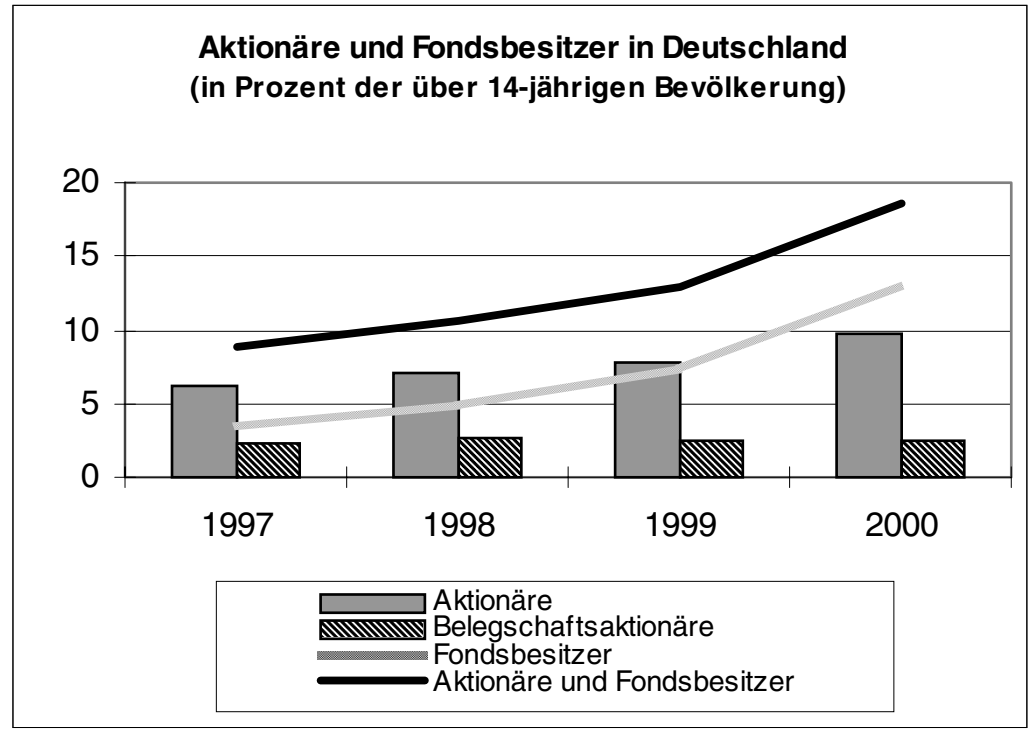

Quelle: DAI 2001

\section{Anteil der Haushalte und Publikumsfonds am Aktienumlauf}

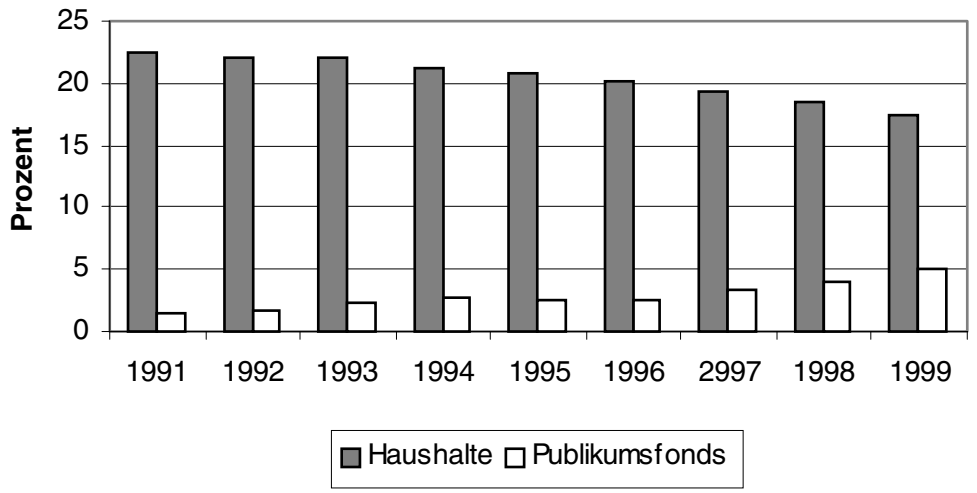

Quelle: DAI 2000: 2

privaten Haushalte am Aktienumlauf. 1999 waren private Haushalte nur zu gut 17\% am gesamten Aktienumlauf in Deutschland beteiligt. Dagegen stieg der Anteil des Auslands sowie der von Investmentfonds (DAI 2000: 2). Zu 
Beginn der 70er Jahre waren Aktien seitens der privaten Haushalte viel begehrter als Mitte der 90er: Im Geldvermögensbestand waren Aktien damals mit einem knapp doppelt so hohen Anteil vertreten (Haushalte ohne Selbständige) (DB 1997: 36). Erst nach dem Börsengang der Telekom 1996 setzte eine Art Aktienbegeisterung bei größeren Teilen der Bevölkerung ein.

Betrachtet man die Aktionärsstruktur in Deutschland, dann zeigt sich die dominante Stellung der Unternehmen, die 1999 noch über 29\% der Anteile halten, vor Banken und Versicherungen mit zusammen 23,5\% und den privaten Haushalten mit 17,5\%. Der Anteil der privaten Haushalte ist in den 90er Jahren gesunken, während der traditionell sehr hohe Anteil der Unternehmen, überwiegend Beteiligungsbesitz, gestiegen ist (der in Tab. 1 dargestellte geringe Anteil der privaten Haushalte für das Jahr 1995 würde auf 20,6\% steigen, rechnete man - wie 1999 - die Selbständigen den privaten Haushalten zu; entsprechend geringer würde dann 1995 der Unternehmensanteil ausfallen). Die Bundesbank schätzt, daß der Aktienbesitz der Unternehmen zu 75\% Beteiligungsbesitz ist, der meist von Aktien-Holdings gehalten wird (DB 1997: 30, 39).

\section{Tab. 1: Aktionärsstruktur in Deutschland}

\begin{tabular}{|l|r|c|}
\hline & 1995 & Ende 1999 \\
\hline Private Haushalte* & 14,6 & 17,5 \\
\hline Unternehmen & 42,1 & 29,3 \\
\hline Staat & 4,3 & 1,0 \\
\hline Banken & 10,3 & 13,5 \\
\hline Versicherungen & 12,4 & 9,0 \\
\hline Investmentfonds & 11,7 & 13,6 \\
\hline Übrige Welt & 8,7 & 16,0 \\
\hline
\end{tabular}

* 1999 inkl. Organisationen ohne Erwerbszweck; ** 1999 inkl. Baussparkassen Quelle: DAI 2000: 2; DB 1997: 29

In dieser Struktur zeigt sich die für Deutschland typische „corporate governance" mit dem vergleichsweise geringen Aktienbesitz der privaten Haushalte, der starken Position der Unternehmen und Banken - im Vergleich zu den USA: Dort ist der Anteil der Unternehmen und vor allem der Banken (Trennbankensystem) sehr gering, während die Pensionsfonds und Versicherungen große Anteile besitzen (vgl. Tab. 2). In den Angaben spiegelt sich die Finanzierungsstruktur deutscher Unternehmen wider: Seit Jahrzehnten, so die Bundesbank, liege der Anteil der Aktien an den gesamten Finanzierungsmitteln der Produktionsunternehmen (Unternehmen ohne Banken und Versicherungen) bei nur etwa 27\%, während fast die Hälfte auf Bankkredite entfällt (DB 1997: 31). 
Tab. 2: Aktienbesitz im internationalen Vergleich (Ende 1995)

\begin{tabular}{|l|l|l|l|l|l|}
\hline & USA & Japan & Deutschland & Frankreich & Großbritannien \\
\hline Private Haushalte & 36,4 & 22,2 & 14,6 & 19,4 & 29,6 \\
\hline Unternehmen & 15,0 & 31,2 & 42,1 & 58,0 & 4,1 \\
\hline Staat & 0,0 & 0,5 & 4,3 & 3,4 & 0,2 \\
\hline Banken & 0,2 & 13,3 & 10,3 & 4,0 & 2,3 \\
\hline Versicherungen, Pensionsfonds & 31,3 & 10,8 & 10,3 & 1,9 & 39,7 \\
\hline Investmentfonds u.ä. & 13,0 & 11,7 & 12,4 & 2,0 & 10,4 \\
\hline Übrige Welt & 4,2 & 10,3 & 8,7 & 11,2 & 13,7 \\
\hline Summe & 100 & 100 & 100 & 100 & 100 \\
\hline
\end{tabular}

Quelle: DB 1997: 29

Die personelle Verteilung des Nettogeld- und Nettoimmobilienvermögens aller privaten Haushalte (also Reinvermögen ohne Gebrauchsvermögen) für das Jahr 1993 zeigt eine deutliche Konzentration bei dem oberen Dezil der Haushalte: Während diese knapp 41\% des gesamten Vermögens (in dieser Abgrenzung) besaßen, verfügten die unteren fünf Dezile über nicht mehr als 5,6\% des gesamten Reinvermögens aller privaten Haushalte (vgl. Tab. 3). Man kann vermuten, daß die Vermögenskonzentration in den 90er Jahren infolge der Aktienhausse und der anhaltenden Einkommensumverteilung zugunsten oberer Schichten kräftig gestiegen ist. Insofern ist das Fazit einer Zwei-Drittel-Gesellschaft (Richard Hauser) zutreffend: Das untere Drittel besitzt überhaupt kein Vermögen, das mittlere Drittel ein kleines, das obere Drittel ein großes Vermögen (Hauser 1999).

Tab. 3: Verteilung der Summe des Nettogeld- und Nettoimmobilienvermögens aller Haushalte 1993 (Westdeutschland)

\begin{tabular}{|l|l|l|}
\hline $\begin{array}{l}\text { Private Haushalte nach der } \begin{array}{l}\text { Summe des Nettovermögens in } \\
\text { aufsteigender Reihenfolge }\end{array} \\
\text { 1. Dezil }\end{array}$ & $\begin{array}{l}\text { Anteil am gesamten Nettogeld- } \\
\text { und Immobilienvermögen in } \\
\text { Prozent }\end{array}$ & $\begin{array}{l}\text { Arithmetisches Mittel des } \\
\text { Vermögens je Haushalt in } \\
\text { DM }\end{array}$ \\
\hline 2. Dezil & $-0,3$ & \\
\hline 3. Dezil & 0,3 & -7.460 \\
\hline 4. Dezil & 0,7 \\
\hline 5. Dezil & 1,6 \\
\hline 6. Dezil & 3,3
\end{tabular}$\quad$\begin{tabular}{l}
6.722 \\
\hline 7. Dezil
\end{tabular}

Quelle: Hauser 1999

Was die Verteilung des Produktivvermögens angeht, so ist die statistische Datenbasis noch schlechter als bei den anderen Vermögensarten (siehe Bohnet 
1999). Es geht dabei um das gewerbliche Anlagevermögen aller Unternehmen aller Rechtsformen, gleich wie es finanziert ist. Krelle u.a. hatten 1968 in einer Aufsehen erregende Pionierstudie errechnet, daß 1,7\% aller privaten Haushalte 70 bis 74\% des Produktivvermögens besitzen (Krelle u.a. 1968). Seitdem gibt es nur einige Schätzungen aus den 70er Jahren. So kommen Mierheim/Wicke für das Jahr $1973 \mathrm{zu}$ folgendem Ergebnis: Auf 0,5\% aller Haushalte entfallen 40\% des volkswirtschaftlichen Produktivvermögens (vgl. Tab. 4). Daß die Vermögenskonzentration im Laufe der 80er und 90er Jahre Jahre noch weiter zugenommen hat, kann man vermuten: Aktienhausse, Unternehmenskonzentration, hohe Realzinsen und eine sinkende Lohnquote könnten dazu beigetragen haben; es mangelt jedoch an verläßlichen empirischen Angaben (vgl. u.a. Schlomann 1993, Braun 2000, Müller 2000).

\section{Tab. 4: Die Verteilung des Produktivvermögens in Deutschland 1973}

\begin{tabular}{|l|l|l|l|}
\hline $\begin{array}{l}\text { Nettovermögen der } \\
\text { Haushalte in DM }\end{array}$ & $\begin{array}{l}\text { in Prozent aller } \\
\text { Haushalte }\end{array}$ & $\begin{array}{l}\text { Anteil am Pro- } \\
\text { duktivvermö- } \\
\text { gen in Prozent }\end{array}$ & $\begin{array}{l}\text { Kumulierter Anteil am Produk- } \\
\text { tivvermögen in Prozent }\end{array}$ \\
\hline bis 10.000 & 30,1 & 1,0 & 1,0 \\
\hline 10.000 bis 100.000 & 45,87 & 8,4 & 9,4 \\
\hline 100.000 bis 2,5 Mio. & 23,57 & 50,6 & 60,0 \\
\hline über 2,5 Mio. & 0,46 & 40,0 & 100,0 \\
\hline
\end{tabular}

Quelle: Mierheim/Wicke 1978, S. 68 f., 99 und 103, eigene Berechnungen

Die Vermögensbildung der Gruppe der mittleren Arbeitnehmerhaushalte hat sich in den letzten Jahrzehnten ganz deutlich auf folgende Präferenzen konzentriert: Wohneigentum und Lebensversicherung als ergänzende Altersvorsorge, zudem Ansparen auf Gebrauchsvermögen (vgl. auch Priewe/Havighorst 1999: 8ff.).

Tab. 5: Einkommen aus Geldvermögen (nach Abzug von Konsumentenkreditzinsen) nach Haushaltsgruppen 1991-98 in DM pro Jahr

\begin{tabular}{|l|l|l|l|l|l|l|l|}
\hline $\begin{array}{l}\text { Haushalts- } \\
\text { typ }\end{array}$ & $\begin{array}{l}\text { Selbstän- } \\
\text { dige }\end{array}$ & Angestellte & Beamte & Arbeiter & $\begin{array}{l}\text { Ar- } \\
\text { beits- } \\
\text { lose }\end{array}$ & Rentner & Pensionäre \\
\hline 1991 & 9.400 & 4.400 & 4.700 & 2.400 & 1.400 & 3.900 & 3.800 \\
\hline 1992 & 10.400 & 5.100 & 5.200 & 2.600 & 1.600 & 4.300 & 4.300 \\
\hline 1993 & 10.500 & 5.100 & 5.100 & 2.500 & 1.600 & 4.300 & 4.400 \\
\hline 1994 & 10.700 & 5.200 & 5.100 & 2.600 & 1.500 & 4.200 & 4.400 \\
\hline 1995 & 10.800 & 5.100 & 5.000 & 2.500 & 1.400 & 4.200 & 4.400 \\
\hline 1996 & 10.500 & 5.000 & 4.900 & 2.500 & 1.300 & 4.200 & 4.400 \\
\hline 1997 & 11.000 & 5.400 & 5.100 & 2.600 & 1.300 & 4.400 & 4.600 \\
\hline 1998 & 11.300 & 5.500 & 5.100 & 2.700 & 1.200 & 4.500 & 4.500 \\
\hline
\end{tabular}

Quelle: Bedau 1999 
Die Geldvermögensbildung der privaten Haushalte ermöglicht laufende Einkommen, die 1998 zu nicht mehr als 7,9\% zum verfügbaren Einkommen der Haushalte beitrugen (ohne Einkommen aus Unternehmertätigkeit - sogar leicht weniger als 1991: damals waren es 8,1\%) (berechnet nach Bedau 1999). Nach wie vor sind die Vermögenseinkommen je Haushalt recht niedrig, und auch der Anstieg in den 90er Jahren fällt schwach aus, insbesondere wenn man die Steigerung der Lebenshaltungskosten von knapp 20\% (1991-98) berücksichtigt (vgl. Tab. 5).

Freilich: Die Konzentration auf die Durchschnittsangaben trübt den Blick auf relevante Minderheiten, die zu einer deutlichen Differenzierung der Vermögensbildung beigetragen haben. Hierzu gehören vor allem diejenigen, die Mitarbeiterbeteiligungen eingegangen sind.

\section{Mitarbeiterbeteiligungen in Deutschland}

Unter Mitarbeiterbeteiligungen werden meist Kapitalbeteiligungen irgendeiner Art seitens der Mitarbeiter am arbeitgebenden Unternehmen verstanden. Es kann sich dabei auch um Gewinnbeteiligungen handeln, die nicht in bar ausgezahlt werden, sondern im „eigenen“ Unternehmen in Form einer Beteiligung angelegt werden. Je nach Rechtsform des Unternehmens und Zielsetzung bieten sich verschiedene Varianten der Kapitalbeteiligung an. Am einfachsten sind Kapitalbeteiligungen in Aktiengesellschaften, während dies in Personengesellschaften und $\mathrm{GmbHs}$ gesellschaftsrechtlich komplizierter ist. Vielfach wird hier eine stille Beteiligung oder ein festverzinsliches bzw. partiarisches Darlehen (d.h. der Zins ist erfolgsabhängig) gewählt, wodurch freilich nur eine Fremdkapitalbeteiligung entsteht. Die Dominanz dieser Rechtsformen erschwert zweifellos Kapitalbeteiligungen von Mitarbeitern.

Traditionell wurden Mitarbeiterbeteiligungen von beiden Tarifparteien mehrheitlich skeptisch bis ablehnend betrachtet. Die meisten Gewerkschaften präferierten Barlöhne und befürchteten, daß Mitarbeiterbeteiligungen die Tariflohnpolitik behindern, für die meisten Beschäftigten zu riskant seien (Arbeitsplatz- und Vermögensrisiko) und den Arbeitnehmerstatus tendenziell untergraben würden. Statt dessen wurden tarifliche Branchen- oder Regionalfonds präferiert. Umgekehrt befürchteten viele Unternehmer Einflußschneisen der Belegschaften und Gewerkschaften hinsichtlich der Verfügungsgewalt der Kapitaleigner bzw. des Managements; zudem wurde die Mobilisierung von Arbeitnehmerkapital meist gar nicht benötigt, weil andere Optionen bestanden. Nach und nach wurden diese Positionen von beiden Tarifparteien zugunsten pragmatischer Lösungen verlassen, wobei die Personalpolitik der Großunternehmen vermutlich den Ausschlag gab, die in starkem Maße auf Belegschaftsaktienprogramme und ähnliche übertarifliche Gratifikationen setzte. Diese gehören heute zum Kernbestand moderner Personalpolitik, zu- 
mindest in größeren und gutsituierten Unternehmen mit einem größeren Anteil hochqualifizierter Mitarbeiter. Die Motive der Unternehmen sind dabei vielfältig: Hoffnung auf Motivationsstärkung und Produktivitätssteigerung, Bindung der Mitarbeiter an das Unternehmen, Förderung partnerschaftlicher, kooperativer Unternehmenskultur etc.

\section{Tab. 6: Formen von Mitarbeiterbeteiligungen}

\begin{tabular}{|c|c|}
\hline $\begin{array}{l}\text { Kapitalbeteiligung } \\
\text { - } \quad \text { Eigenkapital } \\
\text { + Stammaktien } \\
\text { + Vorzugsaktien } \\
\text { + Aktienoptionen } \\
\text { + GmbH-Anteile } \\
\text { + Kommanditeinlage } \\
\text { + Genossenschaftsanteil } \\
\text { Eigenkapitalähnliche Einlagen } \\
\text { + stille Gesellschaft } \\
\text { + Genußscheine } \\
\text { Fremdkapitalbeteiligung } \\
\text { + Mitarbeiterdarlehen (festverzinslich o- } \\
\text { der partiarisch) } \\
\text { + Wandelschuldverschreibung }\end{array}$ & $\begin{array}{l}\text { Gewinnbeteiligung } \\
\text { - } \quad \text { Einbehaltener Gewinnanteil } \\
\text { + Anlage im Unternehmen, z.B. } \\
\text { ++ Eigenkapitalanteil } \\
\text { + Anlage außerhalb des Unternehmens } \\
\text { Barausschüttung } \\
\text { + Verwendung für Konsum } \\
\text { + Verwendung zum Sparen, z.B. } \\
\text { ++ Bausparen } \\
\text { ++ Investmentfonds } \\
\text { ++ Lebensversicherung }\end{array}$ \\
\hline
\end{tabular}

Gleichwohl ist die Verbreitung von Mitarbeiterbeteiligungen in Deutschland relativ gering, wenn auch rasch ansteigend. Nach Angaben der AGP (Arbeitsgemeinschaft Partnerschaft in der Wirtschaft, einer Interessenvertretung der Unternehmen mit Mitarbeiterbeteiligungen), die wohl eher den Umfang über- als unterschätzen, gab es 1999 nicht mehr als 2.700 Unternehmen mit 2,3 Mio. Mitarbeitern, die in irgendeiner Weise am Kapital ihres Unternehmens beteiligt waren. Dabei lag die durchschnittliche Kapitalbeteiligung bei nur knapp 11.000 DM. Die Beteiligungen konzentrieren sich vornehmlich auf Belegschaftsaktien, meist in börsennotierten lukrativen AGs.

Demnach sind nicht mehr als 6,8\% aller Arbeitnehmer in Deutschland am arbeitgebenden Unternehmen beteiligt; und deren Anteil am Produktivvermögen macht nur 0,6\% des gesamtwirtschaftlichen Produktivvermögens aus. Diese Quoten sind auch im internationalen Vergleich sehr gering. Allerdings sind die Daten nicht gesichert. ${ }^{3}$ 
Tab. 7: Verbreitung von Mitarbeiterbeteiligungen in Deutschland 1999

\begin{tabular}{|l|l|l|l|l|}
\hline Beteiligungsform & $\begin{array}{l}\text { Zahl der Un- } \\
\text { ternehmen }\end{array}$ & $\begin{array}{l}\text { Beteiligungs- } \\
\text { wert/ Kurswert } \\
\text { in Mrd. DM }\end{array}$ & $\begin{array}{l}\text { Beteiligte } \\
\text { Mitarbeiter }\end{array}$ & $\begin{array}{l}\text { Beteiligungswert } \\
\text { je Mitarbeiter } \\
\text { in DM }\end{array}$ \\
\hline Belegschaftsaktion & 400 & 21,9 & 1.800 .000 & 12.167 \\
\hline GmbH-Anteile & 150 & 0,05 & 5.000 & 10.000 \\
\hline Genußrechte & 300 & 1,5 & 100.000 & 15.000 \\
\hline Stille Beteiligungen & 650 & 0,355 & 200.000 & 1.775 \\
\hline Indirekte Beteiligungen & 400 & 0,345 & 80.000 & 4.313 \\
\hline Genossenschaftsanteile & 300 & 0,05 & 15.000 & 3.333 \\
\hline Mitarbeiterdarlehen & 500 & 0,8 & 100.000 & 8.000 \\
\hline Summe & 2.700 & 25,0 & 2.300 .000 & 10.870 \\
\hline
\end{tabular}

Quelle: Arbeitsgemeinschaft Partnerschaft in der Wirtschaft, zit. n. Tofaute 1998: 378, ISA 2000: 52

In einzelnen Unternehmen - meist aus dem Kreis der großen börsennotierten Aktiengesellschaften - erreichen jedoch Mitarbeiterbeteiligungen einen beachtlichen Umfang. Herausragend in Deutschland ist die Siemens AG. 21\% des Stammkapitals wird von Mitarbeitern gehalten, die sogar erwägen, ihre Beteiligungen aufzustocken, um eine Sperrminorität gegen feindliche Übernahmen zu erlangen. Seit 1967 werden Belegschaftsaktien mit erheblichen Preisabschlägen angeboten, für übertariflich bezahlte Mitarbeiter in besonders großem Umfang. Häufig liegt der Vorzugspreis bei 50\% des Börsenkurses. 82\% der Belegschaft hat von dem Angebot Gebrauch gemacht, darunter 48\% der gewerblichen Mitarbeiter (vgl. die Übersicht bei ISA 2000a). Zahlreiche Großunternehmen - so z.B. DaimlerChrysler, Volkswagen, Schering, Continental, Bertelsmann, Deutsche Telekom - haben ähnliche Programme, auch bei vielen kleineren und mittleren Unternehmen breiten sich Mitarbeiterbeteiligungsmodelle der verschiedensten Art rasch aus (vgl. BMAS 1997 und 1998, ISA 2000a, Müller 2000). Gefördert wird diese Tendenz auch durch die Reform der Vermögensbildung in Arbeitnehmerhand seit 1999, welche die Beteiligung am Produktivkapital erstmals massiv unterstützt. In den Neugründungen der „New Economy“ liegt der Anteil derjenigen Mitarbeiter, die mit Aktienoptionen u.ä. am Unternehmenskapital beteiligt sind, Berichten der Wirtschaftspresse zufolge bei $70 \%$. Hier dürfte es sich einerseits um besonders qualifizierte und knappe Mitarbeiter handeln, andererseits auch um defizitäre Firmen mit geringem Eigenkapital und erheblichen Liquiditätsproblemen; hohe Risiken und hohe Chancen dürften eng gekoppelt sein. Vor einer Verallgemeinerung dieses sehr speziellen Firmentyps kann man nur warnen.

In anderen OECD-Ländern ist der Umfang von Kapitalbeteiligungen der Mitarbeiter erheblich größer als in Deutschland, insbesondere in den USA (Priewe/Havighorst 1999: 14ff.), aber auch in Großbritannien und in Frankreich existieren vergleichsweise starke Beteiligungen. In den USA ist diese 
Praxis nahezu ausschließlich durch betriebliche Altersvorsorge bedingt. Infolge der schwachen staatlichen Rentenvorsorge ist die betriebliche Säule der Altersvorsorge bei den „besserverdienenden“ Arbeitnehmern stärker als in Deutschland ausgebaut, meist in Form von ESOPs: Employee Stock Ownership Plans. Ein Unternehmen gründet einen Fonds, der treuhänderisch verwaltet wird und Kredite aufnimmt, um Aktien des Unternehmens oder anderer Unternehmen zu kaufen; dabei gibt das Unternehmen eine Bürgschaftserklärung für den ESOP ab. Das Unternehmen schießt jährlich steuerlich abzugsfähige Bareinlagen in den ESOP ein, um Zins und Tilgung zu finanzieren. Der Treuhänder bucht die Wertpapiere auf die individuellen Konten der Arbeitnehmer um. Im Grunde handelt es sich um die Anlage von Pensionsrückstellungen. Nach einer bestimmten Sperrzeit (nach 10 Jahren oder ab dem 55. Lebensjahr) kann ein Teil des Anlagevermögens zwecks Diversifizierung des Portfolios seitens des Arbeitnehmers umgeschichtet werden. Neben den ESOPs existieren sog. 401k-Pläne, steuerlich begünstigte Investmentfonds auf Unternehmensebene, und Granted Stock Options (GSO), Optionspläne für Mitarbeiter. Rund 15\% aller Erwerbstätigen in den USA sind in einer der drei Formen am Produktivkapital beteiligt (ISA 2000: 113). Hier fungiert die Altersvorsorge als Motor der Beteiligung der Arbeitnehmer am Produktivvermögen - dem stehen freilich, im Gegensatz zu Deutschland, viel geringere Rentenansprüche an die staatliche Rentenversicherung gegenüber. Ähnliche Konstruktionen existieren in Großbritannien, jedoch sind sie weniger auf die Altersvorsorge, sondern auf ertragsabhängige Gewinnbeteiligungen ausgerichtet.

In Frankreich dominieren hingegen staatlich vorgeschriebene Beteiligungen der Mitarbeiter am Gewinn (in festgelegtem Umfang, ab einer Mindestgröße der Unternehmen), die im Unternehmen oder außerhalb für eine bestimmte Sperrzeit angelegt werden müssen. Die Altersvorsorge steht hier nicht im Vordergrund (Priewe/Havighorst 1999: 20ff.). Von den beiden relevantesten Beteiligungsformen (intéressement und participation) profitierten zu Beginn der 90er Jahre 19\% bzw. gut 30\% der Arbeitnehmer (außerhalb des öffentlichen Dienstes).

Wie groß ist nun der Umfang der Gewinnbeteiligungen? Versteht man darunter mehr oder minder variable betriebliche Sonderleistungen wie Prämien und Gratifikationen, die vorwiegend ertragsabhängig sind und bar ausgezahlt werden, dann läßt sich ein deutlicher Anstieg in den 90er Jahren in Deutschland feststellen (Pannenberg/Schröder 2000). Nach Berechnungen auf der Basis des Sozio-ökonomischen Panels (SOEP) erhielten 1997 knapp 13\% aller abhängig Beschäftigten Sondervergütungen ${ }^{4}$ in Höhe von durchschnittlich

4 Die Abgrenzung der „Sonderleistungen“ im SOEP von Tariflohnbestandteilen bzw. durch übertarifliche betriebliche Vereinbarungen festgelegten Leistungen ist nicht ganz eindeutig. 
DM 5.700 DM oder 6,6\% des direkten Entgelts (Ostdeutschland: 8,3\% der Beschäftigten, 2.149 DM bzw. 4,2\% des direkten Entgelts) (vgl. Tab. 8). Je höher die Einkommen, desto größer ist der Anteil variabler Sondervergütungen. Aber selbst im oberen Viertel der Einkommensgruppen bezogen 1997 nicht mehr als gut 24\% derartige Leistungen, die nur 8\% der direkten Entgelte ausmachten. Auch ist nicht ganz klar, wie variabel die Sonderleistungen sind bzw. ob sie nicht zu quasi-festen Einkommensbestandteilen geworden sind, wie dies beispielsweise über viele Jahre hinweg in Japan der Fall war: Der dortige hohe Anteil der Bonus-Zahlungen war bis in die jüngste Zeit ein fester Entgeltbestandteil (Layard u.a.1991: 500f).

Tab. 8: Betriebliche Sondervergütungen in Deutschland 1991-97

\begin{tabular}{|l|l|l|l|l|l|l|l|}
\hline & 1991 & 1992 & 1993 & 1994 & 1995 & 1996 & 1997 \\
\hline Anteil der abhängig Beschäftigten mit jährlichen Sondervergütungen in Prozent \\
\hline West & 11,8 & 11,4 & 10,9 & 11,2 & 12,3 & 12,9 & 12,9 \\
\hline Ost & 5,6 & 6,5 & 7,0 & 7,3 & 5,4 & 8,0 & 8,3 \\
\hline Höhe der jährlichen Sondervergütung in DM \\
\hline West & 5.450 & 5.650 & 4.179 & 4.943 & 4.885 & 5.431 & 5.708 \\
\hline Ost & 1.217 & 2.197 & 2.548 & 2.187 & 2.553 & 2.244 & 2.149 \\
\hline Anteil der Sondervergütung am direkten Entgelt in Prozent & \multicolumn{1}{l|}{} \\
\hline West & 5,9 & 6,4 & 5,2 & 6,1 & 5,9 & 6,0 & 6,6 \\
\hline Ost & 3,6 & 5,1 & 5,0 & 4,4 & 6,0 & 4,4 & 4,2 \\
\hline Anteil abhängig Beschäftigter mit Sondervergütungen nach Einkommensquartilen in Prozent \\
\hline bis $25 \%$ & 3,5 & 3,7 & 4,4 & 5,4 & 5,2 & 6,7 & 6,2 \\
\hline 25 bis $50 \%$ & 4,8 & 6,4 & 6,2 & 6,6 & 5,0 & 5,2 & 6,5 \\
\hline 50 bis 75\% & 12,7 & 11,0 & 11,4 & 10,0 & 10,9 & 12,2 & 12,1 \\
\hline über 75\% & 21,6 & 21,6 & 19,8 & 20,5 & 24,4 & 24,4 & 24,1 \\
\hline Anteil der Sondervergütung am direkten Entgelt nach Einkommensquartilen in Prozent \\
\hline bis 25\% & 4,0 & 6,2 & 3,7 & 3,4 & 5,5 & 4,4 & 4,7 \\
\hline 25 bis 50\% & 3,2 & 4,3 & 2,9 & 4,2 & 2,6 & 3,0 & 5,1 \\
\hline 50 bis 75\% & 5,3 & 4,5 & 5,3 & 4,8 & 5,2 & 4,6 & 4,4 \\
\hline über 75\% & 6,7 & 7,8 & 6,2 & 7,6 & 7,1 & 7,5 & 8,1 \\
\hline
\end{tabular}

Quelle: Pannenberg/Schröder 2000

Inzwischen hat sich das „Bündnis für Arbeit“ mit einer Erklärung von BDA und DGB für Gewinnbeteiligungen und Lohnflexibilisierung ausgesprochen, mit ausdrücklichem Verweis auf erhoffte Beschäftigungswirkungen ${ }^{5}$. Seit langem argumentiert die neoklassisch ausgerichtete wirtschaftspolitische Beratung in allen westlichen Ländern in diese Richtung („profit-sharing" ist für viele seit Weitzman (1987) geradezu eine Zauberformel), jedoch gibt es massive theoretische Bedenken und eine Vielzahl von empirischen Untersuchungen, die keine eindeutigen Zusammenhänge identifizieren konnten (vgl. Priewe/

5 Gemeinsame Erklärung von BDA und DGB vom 6.7.1999 anläßlich des 3. Gesprächs „Bündnis für Arbeit“. 
Havighorst 1999: 44ff., 61ff.). Nicht einmal ein kausaler Zusammenhang von Gewinnbeteiligung und Produktivitätssteigerung ist mit hinreichender Sicherheit nachweisbar, geschweige denn ein Bezug zur Beschäftigung. In Ländern mit dezentraler Lohnfindung, insbesondere in Großbritannien, hat sich „profit related pay“ am stärksten durchgesetzt, allerdings liegt der Anteil am Festlohn mit 2\% - 5\% (für das Jahr 1993) sehr niedrig. Insgesamt ist in den OECD-Ländern eine flexible, wenigstens teilweise ertragsabhängige Entlohnung nur ganz schwach verbreitet - die Arbeitnehmer sind eben stark risikoavers und präferieren andere Entgeltformen (vgl. den Survey in OECD 1995 und Priewe/Havighorst 1999).

\section{Die ökonomische Logik von Kapital- und Gewinnbeteiligungen der Arbeitnehmer}

Auch wenn der bislang empirisch feststellbare Umfang von Kapital- und Gewinnbeteiligungen von Arbeitnehmern sowie das Ausmaß von Lohnflexibilisierung sich offenbar nicht nur in Deutschland in engen Grenzen halten, wenngleich überall Veränderungstendenzen zu beobachten sind, stellt sich die Frage, ob es überzeugende ökonomische Gründe dafür gibt, daß sich dies mittel- bis langfristig grundlegend ändern könnte.

\section{Shareholder-Value Orientierung}

Wie wirkt die Shareholder-Value-Orientierung auf die Kapitalbeteiligung der Mitarbeiter? Eigentlich ist die Idee, Mitarbeiter auf das Ziel der Maximierung des Unternehmenswertes per Optionsplan zu motivieren, auf das Management beschränkt. Vielfach wurden jedoch Optionspläne und ähnliche Programme auf alle leitenden Mitarbeiter, mitunter auf die ganze Belegschaft, mit graduellen Abstufungen, angewendet. Die Grundkonzeption beruht auf der institutionenökonomischen Agency-Theorie; sie konstatiert einen Interessenwiderspruch zwischen Kapitaleignern und Management, ähnlich wie bereits Berle und Means in den 30er Jahren (vgl. ausführlich Winter 2000: 5ff.). Da Eigentum und Kontrolle auseinanderfielen, würde das Management zu stark eigene Interessen verfolgen, an Macht- und Prestigeausweitung, GröBenwachstum und consumption on the job mehr Interesse haben als an der Vermehrung des Eigenkapitals der Aktionäre, moral hazard entstünde, die Risikoaversion des Managements, die durch das unternehmensspezifische $\mathrm{Hu}$ mankapital bedingt sei, würde sich gegenüber den Interessen der Aktionäre durchsetzen. Zudem seien die Möglichkeiten zum Firmenvergleich für die Aktionäre hinsichtlich der Eigenkapitalrentabilität und damit die Kapitalmobilität eingeschränkt. Herkömmliche Gewinnbeteiligungssysteme, auf Kennziffern aus dem Rechnungswesen - z.B. dem Jahresüberschuß - beruhend, seien unzulänglich (statischer Charakter, fehlende Berücksichtigung von 
Zeitpräferenzen, Risikopräferenzen würden ausgeklammert, fehlende Vergleichsmaßstäbe etc.). Der Vorteil von Optionsplänen bestünde darin, die Zielkonflikte zwischen Managment und Kapitaleignern zu vermindern, indem das Management selbst am Kapital beteiligt und dessen Risikobereitschaft erhöht wird.

Hier ist nicht der Ort, den Shareholder-Value-Ansatz kritisch zu bewerten (vgl. u.a. Bischoff 1994 sowie den Beitrag von Sablowski/Rupp in diesem Heft). Dennoch seien eine Reihe von kritischen Einwänden formuliert, die aufzeigen, daß das Konzept alles andere als konsistent und ausgereift ist:

(1) In den meisten Ansätzen erfolgt die Bewertung des Eigenkapitals, also der diskontierten zukünftigen cash flows, durch den Börsenkurs. Entweder wird ein perfekter, vollkommener Kapitalmarkt unterstellt, den es freilich in der Realität nicht gibt, oder es wird ein Maßstab gewählt, der infolge seiner Volatilität kaum als Richtschnur der Unternehmnenspolitik geeignet ist. Obwohl gerade eine Langfristorientierung intendiert ist (eben die Maximierung diskontierter zukünftiger cash flows), tritt eine Kurzfristorientierung ein, wenn der aktuelle Börsenwert als Maßstab dient. Eine pragmatische Lösung läßt sich auch nicht erzielen, wenn bestimmte Vergleichswerte (z.B. Börsenkurse der jeweiligen Branche, DAX- oder CDAX-Entwicklung) als Maßstab gewählt werden.

(2) Der Börsenkurs kann nur teilweise vom Management selbst beeinflußt werden. In hohem Maße, das jedoch nicht quantifizierbar ist, ist er von makroökonomischen Faktoren, Erwartungsänderungen, von der Geldpolitik etc. abhängig. Schon gar nicht existiert eine individuelle Zurechenbarkeit von Manager-Leistung und Börsenentwicklung.

(3) Je nach Art der Optionspläne kann eine sehr starke Risikoorientierung des Managements induziert werden - die Risikohaftung ist begrenzt, die Chancen sind unbegrenzt.

(4) Manipulationsgefahren und Insiderprobleme sind erheblich.

Vielfach sind die Optionspläne auch außerordentlich teuer, sie haben mitunter zu einer enormen Überbezahlung des Managements geführt. Es scheint, als ob das Management im Principal-Agent-Konflikt häufig am längeren Hebel sitzt und die Grundidee so ausformt, daß das Ganze zu seinen Gunsten ausgeht. Die Verfechter des Shareholder-Value-Gedankens betrachten diese Einwände als lösbare Ausgestaltungsprobleme (vgl. Winter 2000: 48ff.). Dem ist entgegen zu halten, daß die Lösung nur im Rahmen einer effizienten corporate governance gefunden werden kann, die sich selbst in raschem Wandel befindet. Eine ausgereifte Konzeption, die sich mit überlegener ökonomischer Logik allerorten durchsetzen wird und zu einer starken Beteiligung des Managements und breiterer Arbeitnehmerschichten führt, ist noch längst nicht zu erkennen. 


\section{Lohnflexibilisierung und Gewinnbeteiligungen}

Zweifellos sind die Unternehmen an Vergütungssystemen interessiert, die einerseits produktivitäts- und motivationsfördernde Anreize setzen, ihnen andererseits aber auch je nach Marktlage zusätzliche Flexibilitätsspielräume geben. Unter den Bedingungen von Flächentarifverträgen bestehen in den Unternehmen mit überdurchschnittlicher Produktivitätsentwicklung übertarifliche Verteilungsspielräume, die mit Gewinn- und Kapitalbeteiligungen genutzt werden können. Für die restlichen Unternehmen ist dies viel schwieriger. Das Problem von ertragsabhängigen Lohnbestandteilen besteht vor allem darin, daß kein direkter Bezug zur individuellen Leistung existiert. Insofern bleiben die erhofften Effizienzlohneffekte immer unsicher.

Sollte in großem Stil von festen Tariflöhnen im Flächentarifvertrag abgegangen werden zugunsten niedriger Fixlöhne und ertragsabhängiger Zusatzlöhne, so ist mit erheblichem Widerstand der Arbeitnehmer zu rechnen, die nach wie vor eher risikoavers sind - was ihrer Vermögenslage auch eher entspricht. Entsteht jedoch ein Lohnbildungssystem, daß die Unternehmen mit schlechter performance (bei Produktion, Produktivität, Gewinnen) durch niedrige Löhne belohnt und spiegelbildlich die überlegenen Unternehmen bestraft, so werden geradezu Fehlanreize gesetzt, die produktivitätshemmend wirken und das Wachstum der Einkommen (auch der Gewinne) drücken. Gleichwohl erscheint aus der mikroökonomischen Perspektive der einzelnen Unternehmen eine derartige Lohnpolitik rational.

Allerdings können bei einem Lohnsystem mit hohem Anteil von Bonuszahlungen bei geringem Festlohn gravierende makroökonomische Probleme auftreten. Ein solches System wirkt nämlich prozyklisch, inflations- und deflationsverstärkend: In Phasen guten Konjunktur steigen die Lohnkosten (dies gilt auch dann, wenn die Bonuszahlungen als Gewinnbeteiligung deklariert werden) überproportional, denn die Amplitude der Gewinndynamik ist im allgemeinen viel stärker als die des Wirtschaftswachstums oder der Produktivität; umgekehrt sinken die Lohnkosten in schwachen Konjunkturphasen, wodurch bei zunächst starren Preisen die reale Konsumnachfrage geschwächt würde, längerfristig aber das Preisniveau sinken würde. Während eine produktivitätsorientierte Lohnpolitik, bezogen auf die gesamtwirtschaftliche Lohnentwicklung, wie ein Anker des Preisniveaus wirkt, destabilisieren flexible Löhne das Preisniveau, nach oben wie nach unten. Freilich gilt dies nur dann, wenn die Lohnflexibilisierung solche Ausmaße angenommen hat, dass sie gesamtwirtschaftlich relevant ist.

Von den verschiedenen Protagonisten der Lohnflexibilisierung wird behauptet, daß diese beschäftigungsfördernd wirken würde - denn Arbeitslosigkeit wird aus neoklassischer Sicht ja gerade durch starre Reallöhne ausgelöst. Dabei argumentiert Weitzman, der wichtigste Protagonist der share economy, 
im Rahmen eines gesamtwirtschaftlichen Modells der neoklassischen Synthese (IS-LM-Modell mit Realkasseneffekten und monopolistischer Konkurrenz auf den Gütermärkten) mit variablem Preisniveau. Deflationäre Tendenzen werden als beschäftigungsstabilisierend verstanden (Weitzman 1987, kritisch dazu Priewe/Havighorst 1999: 61ff., Rothschild 1986/87). Im Unterschied dazu argumentiert Sinn im Rahmen traditioneller neoklassischer Annahmen, wobei er ein konstantes Preisniveau, bestimmt durch die Geldmenge, unterstellt (Sinn 1997). Er plädiert dafür, „Arbeitsplatzbesitzern“ einen äquivalenten Tausch von Lohnbestandteilen gegen Kapitalbeteiligungen am eigenen Unternehmen anzubieten, während für Neueinstellungen der abgesenkte Lohn ohne Kapitalbeteiligung gelten solle. Solange der Reallohn unter dem Wertgrenzprodukt der Arbeit liegt, würden die Unternehmen mit Neueinstellungen reagieren. Mangel an Güternachfrage sieht Sinn nicht, der Kapitalstock könne selbst kurzfristig mit mehr Arbeit kombiniert werden. Derartige Modelle bleiben im Rahmen rigider neoklassischer Modellprämissen, die vielfach kritisiert wurden (vgl. dazu Priewe/Havighorst 1999; 70ff.). Realistisch erscheint die Einschätzung von Layard u.a. (1991: 502), daß profit sharing eher zu Produktivitätssteigerungen führt, kaum aber zu Beschäftigungszunahmen. ${ }^{6}$

Insgesamt ist keine allgemeine Marktlogik erkennbar, die zu durchschlagenden Erfolgen von Lohnflexibilisierung im Sinne von Gewinn- und/oder Kapitalbeteiligungen führt. Einleuchtend ist, daß in den Unternehmen mit überdurchschnittlicher ökonomischer performance wenigstens ein Teil der Mitarbeiter mit ertragsabhängigen Gratifikationen entgolten wird, um die Effizienz der Arbeit zu steigern, die Bindung an das Unternehmen zu erhöhen, betriebsspezifische Qualifikationen zu sichern und um eine sozialpartnerschaftliche Unternehmenskultur zu pflegen. Daß die Entlohnung von abhängiger Arbeit eines Tages so ähnlich wie etwa die von Handelsvertretern gestaltet wird, nämlich mit einem niedrigen Fixum und einem großen erfolgsabhängigem Bestandteil, so die Vision von Weitzman, ist nicht erkennbar. Wäre es so, würden die Arbeitnehmer zu quasi-Selbständigen mutieren. Entscheidend sind freilich die Interessen und der Widerstand der Beschäftigten. Wie dargestellt ist das Ausmaß der Lohnflexibilisierung in den meisten OECD-Ländern gering geblieben, selbst bei schwachem gewerkschaftlichem Organisationsgrad. Je höher das Qualifikations- und das Einkommensniveau, desto eher ist mit der Akzeptanz von flexiblen Löhnen zu rechnen.

6 Genau genommen müßte bei näherer Betrachtung zwischen Beschäftigungsstabilisierung bei konjunkturellen Schwankungen und davon unabhängiger dauerhafter Beschäftigungssteigerung unterschieden werden. 


\section{Kapitalbeteiligungen zur Altersvorsorge}

Hier besteht kein Zweifel, daß Arbeitnehmer ähnlich wie Selbständige in Zukunft stärker private oder betrieblich organisierte Altersvorsorge betreiben werden, indem sie u.a. über den Kauf von Wertpapieren Kapitalbeteiligungen eingehen. Dabei ist allerdings zu berücksichtigen, daß Mitarbeiterbeteiligungen am arbeitgebenden Unternehmen in aller Regel schlechte Varianten der Altersvorsorge sind: zu wenig Risikostreuung, keine Absicherung von Langlebigkeit oder Invalidität. Hierzu bedarf es spezieller Anlageformen, wenn allzu große Risiken vermieden werden sollen. Aber selbst dann verblieben jene makroökonomischen Risiken, die mit kapitalgedeckten Verfahren der Alterssicherung verbunden sind, wenn diese in großem Stil eingeführt werden: keine Inflationssicherung, Instabilität der Wertpapierpreise, insbesondere im Falle des Verkaufs, Konsumschwächung in der Ansparphase. Überdies ist zu prüfen, ob Vorsorgesparen zusätzliches Sparen impliziert oder nur eine Umschichtung der Sparaufwendungen bewirkt. Im letzteren Fall treten die erwähnten Probleme in der Ansparphase zwar nicht auf, aber möglicherweise ergibt sich keine zusätzliche Beteiligung der Arbeitnehmer am Produktivvermögen (je nachdem welche Anlageformen in das Portfolio einbezogen werden). Mithin ist auch hier wohl keine stürmische Zunahme der Kapitalbeteiligungen von Arbeitnehmern auf absehbare Zeit zu erwarten.

\section{Fazit}

Die Schlußfolgerungen aus der Bestandsaufnahme der eingangs geschilderten Trends sind ziemlich eindeutig:

- Von einer Verbesserung der Vermögens-, insbesondere der Produktivvermögensverteilung in Deutschland in den 90er Jahren zugunsten der Arbeitnehmer kann keine Rede sein. Das Volk der Aktionäre oder der Teilhaber ist eine Schimäre. Hinter der Zunahme der Zahl der Aktionäre verbirgt sich eine starke Vermögenspolarisierung. Fast die Hälfte der privaten Haushalte ist vermögenslos.

- Daß im Zuge der Shareholder-Value-Philosophie eine nachhaltige Änderung der Vermögensverteilung eintritt, hängt sehr stark von der inneren Konsistenz, Ausgestaltung und Durchsetzbarkeit dieser Philosophie ab. Die Euphorie der Befürworter erscheint weit überzogen. In den 90er Jahren hat sich allerdings infolge dieser Orientierung und der spekulativen Überhöhung der Wertpapierpreise eine deutliche Umverteilung zugunsten der Alt-Vermögensbesitzer ergeben, die vorrangig dem oberen Drittel der privaten Haushalte zuzurechnen sind.

- Von einem gesamtwirtschaftlich relevanten Ausmaß von Mitarbeiterbeteilungen kann in Deutschland nicht die Rede sein. Zudem ist der Umfang der Beteiligungen jener 6-7\% aller Arbeitnehmer außerordent- 
lich gering - unter 1\% des Produktivvermögens.

- Auch das Ausmaß der Gewinnbeteiligungen ist quantitativ hinsichtlich der Höhe und der Zahl der Beteiligten sehr gering, wenn auch leicht steigend.

- Aus Gründen der ergänzenden privaten oder betrieblichen Altersvorsorge wird der Umfang des Wertpapierbesitzes und damit wohl auch der Produktivvermögensbeteiligung der Arbeitnehmer leicht steigen, aber zu einem Teil handelt es sich um eine bloße Umschichtung innerhalb der verschiedenen Sparformen.

- Für eine kleine Gruppe der vorwiegend „besserverdienenden“ Arbeitnehmer (die sicher kleiner als 10\% der abhängig Beschäftigten ist) lassen sich merkliche Tendenzen zu Kapital- und/oder Gewinnbeteiligungen feststellen, die sich wohl in Zukunft noch verstärken werden. Aber selbst in diesen Gruppen bleibt das traditionelle Lohn- und Gehaltseinkommen die bei weitem dominante Entgeltform.

Aus den Proletariern von einst, die nur ihre Ketten zu verlieren hatten, mögen Arbeitnehmer und Mitarbeiter geworden sein - aber ihr Vermögen ist für die Hälfte nur die Arbeitskraft und deren Qualifikation, für den Rest zusätzlich das Wohnungseigentum, eine Lebensversicherung und ein bißchen Geldvermögen, wozu auch ein paar Aktien gehören können. Die Polarisierung der Vermögensverteilung ist ungebrochen. Marx ist auch zu Beginn des 21. Jahrhundert - in dieser Frage - immer noch näher an der Wirklichkeit als Ludwig Erhard mit seiner Teilhaber-Gesellschaft.

\section{Literatur}

Bedau, K.-D., (1999): Geldvermögen und Vermögenseinkommen der privaten Haushalte, in: DIW-Wochenbericht 30/1999.

Bischoff, J., (1994): Das Shareholder Value Konzept, Wiesbaden.

BMAS (Bundesministerium für Arbeit und Sozialordnung)(HG.) (1997): Forschungsbericht: Praktisch erprobte betriebliche Vereinbarungen zur Kapitalbeteiligung der Arbeitnehmer. Endbericht. Bonn.

- (1998): Mitarbeiterbeteiligung am Produktivvermögen. Ein Wegweiser für Arbeitnehmer und Arbeitgeber, Bonn.

Bohnet, A. (1999): Finanzwissenschaft: Grundlagen staatlicher Verteilungspolitik, 2. Auflage, München.

Bundesregierung (1996): Antwort der Bundesregierung auf die Große Anfrage zu „Entwicklung der Vermögen und ihrer Verteilung" vom 28.2.1996, Bonn.

Braun, R. (2000): Vermögensbildung privater Haushalte, Frankfurt/M.

DAI (Deutsches Aktieninstitut) (2000): Aktien und Aktienfonds stark gefragt. Spareinlagen 1999 erstmals rückläufig. DAI-Kurzstudie 3/2000, Frankfurt/M.

- (2001): DAI-Factbook, Stand Januar 2001, Frankfurt/M.

DB (1995), Deutsche Bundesbank: Die Entwicklung des Wertpapierbesitzes in Deutschland seit Ende 1989, in: Monatsberichte, August, S. 57-69.

DB (1997), Deutsche Bundesbank, Die Aktie als Finanzierungs- und Anlageinstrument, in: Monatsberichte, Januar, S. 27-42.

DB (1999), Deutsche Bundesbank, Zur Entwicklung der privaten Vermögensposition seit Beginn der neunziger Jahre, in: Monatsberichte, Januar, S. 33-50. 
DB (2000), Deutsche Bundesbank, Die gesamtwirtschaftlichen Finanzierungsströme im Jahr 1999, in: Monatsberichte, Juni, S. 15-44.

Fuest, W., u.a. (Hg.) (1997): Vermögensbildung in Arbeitnehmerhand, Köln.

Hauser, R. (1999): Große Vermögen, kleine Vermögen und überhaupt kein Vermögen. In: Neue Caritas, Heft 3.

ISA Consult (2000): Einkommen der Zukunft. Beteiligung der Arbeitnehmer/innen am Produktivkapital: Neue Elemente der Einkommensgestaltung für das 21. Jahrhundert (Abschlußbericht), Hannover, Oktober (Manuskript).

ISA Consult (2000a): Fachtagung „Entwicklungsperspektiven der Mitarbeiterbeteiligung - Europäische Initiativen unter PEPPER II“, Hannover.

Krelle, W., u.a. (1978): Überbetriebliche Ertragsbeteiligung der Arbeitnehmer; mit einer Untersuchung über die Vermögensstruktur der Bundesrepublik Deutschland, Bd. 2, Tübingen.

Layard, R., u.a. (1991): Unemployment. Macroeconomic Performance and the Labour Market. Oxford.

Mierheim, H., Wicke, L. (1978): Die personelle Vermögensverteilung, Tübingen.

Müller, U. (2000): Die vermögens- und verteilungspolitischen Aspekte einer Mitarbeiterbeteiligung, Frankfurt/M.

OECD (1995): Employment Outlook, Paris.

Pannenberg, M., Schröder, M. (2000): Betriebliche Sondervergütungen in Deutschland, in: DIWWochenbericht, 35/2000.

Priewe, J., (2000): Mitarbeiterbeteiligung: Wohin führt der Weg? Optionen und Grenzen für die Zukunft, in: ISA-Consult (Hg.), Mitarbeiterbeteiligung als Instrument der Vermögenspolitik und Alterssicherung - Bilanz und Perspektiven, Hannover, S. 27-52.

Priewe, J., Havighorst, F. (1999): Auf dem Weg zur Teilhabergesellschaft? Investivlöhne, Gewinnund Kapitalbeteiligungen der Arbeitnehmer in Westeuropa und den USA - eine vergleichende Bestandsaufnahme, Bonn (Reihe „Wirtschaftspolitische Diskurse“ Nr. 123 der Friedrich-EbertStiftung).

Rothschild, K.W. (1986/87): Is there a Weitzman miracle? In: Journal of Post Keynesian Economics, Vol. IX, No. 2, S. 198 -211.

Schlomann, H. (1993): Die Entwicklung der Vermögensverteilung in Westdeutschland, in: Huster, E.-U. (Hg.), Reichtum in Deutschland. Der diskrete Charme der sozialen Distanz. Frankfurt/M. S. 54-83.

Sinn, H.-W. (1997): Kapitalbeteiligungen und Lohndifferenzierung: ein Vorschlag zur Lösung der Beschäftigungskrise. In: Mitteilungen aus der Arbeitsmarkt- und Berufsforschung, Heft 4, S. $822 \mathrm{ff}$.

SVR (2000): Sachverständigenrat zur Begutachtung der gesamtwirtschaftlichen Entwicklung, Jahresgutachten 2000/1, Bonn.

Tofaute, H. (1998): Arbeitnehmerbeteiligung am Produktivkapital, in: WSI-Mitteilungen, Heft 6, S. $371-380$.

Weitzman, M.L. (1987): Das Beteiligungsmodell. Vollbeschäftigung durch flexible Löhne. Frankfurt/M.

Winter, St. (2000): Optionspläne als Instrument wertorientierter Managementvergütung, Frankfurt/. 